\title{
Effect of Estrus-Al interval on timed Al pregnancy rates in beef cows inseminated with fresh extended or frozen thawed semen
}

\author{
R. K. Kasimanickam* and W. D. Whittier
}

Department of Large Animal Clinical Sciences,

Virginia Polytechnic Institute and State University, Blacksburg, VA 24061, USA

* Corresponding author present address : Department of Veterinary Clinical Sciences, College of Veterinary Medicine, Washington State University, USA, Email: ramkasi@vetemd.wsu.edu Published online at www.veterinaryworld.org on 25-03-2011

\begin{abstract}
We hypothesize that insemination with fresh extended semen will improve the AI pregnancy rate due to its prolonged longevity in female reproductive tract compared to frozen thawed semen. The objective of this trial was to determine the effect of fresh and frozen semen on fixed-time AI pregnancy rate in beef cows synchronized with progesterone based CO-Synch protocols and inseminated at different estrus-AI intervals. Angus cross beef cows ( $\mathrm{N}=180)$ were synchronized with $\mathrm{CO}-$ Synch-CIDR protocols for a fixed-time AI. A subset of cows $(\mathrm{N}=110)$ received Heatwatch estrus detection system's pressure sensor at CIDR removal to determine the time of estrus. Cows were divided into two groups, inseminated either at $47 \mathrm{~h}$ (early) or at $67 \mathrm{~h}$ (late) from CIDR removal with fresh extended ( 3 million sperm) or of frozen thawed (20 million sperm) semen. Results indicated that cows inseminated at $67 \mathrm{~h}$ had numerically higher fixed time AI pregnancy compared to cows inseminated at $47 \mathrm{~h}$ [44.4\% (40/90) vs. 33.3\% (30/90); $\mathrm{P}=0.13$ ]. Cows inseminated with frozen thawed semen had similar fixed time AI pregnancy compared to fresh extended semen [40.8 (31/76) vs. 37.5\% (39/104); P=0.66]. AI-estrus interval was divided into three groups $<0 \mathrm{~h}$ (AI occurred before estrus), 0 to $16 \mathrm{~h}$ and $>16 \mathrm{~h}$ (AI occurred $16 \mathrm{~h}$ after estrus). The AI pregnancy rates for fresh semen for the 3 estrus-AI intervals were similar to frozen semen. In conclusion, the fresh semen yielded similar AI pregnancy as frozen semen when inseminated at different estrus-AI interval in a fixed time AI breeding program in beef cows.

Keywords: Estrus, Artificial Insemination, Synchronization of estrus, Pregnancy, Frozen Semen, Thawed Semen.

\section{I ntroduction}

In cattle, the AI pregnancy rate after insemination with fresh extended semen with lesser sperm numbers in an insemination dose is similar compared to frozen thawed semen with higher sperm numbers in an insemination dose (Vishwanath et al., 1996). This is due to the impairment of functional and structural parameters of sperm imposed by freeze-thaw process (Parks and Graham, 1992). Therefore, sperm after freeze thaw process are less likely either to survive long enough in the female reproductive tract or to maintain competency in fertilization, as compared to fresh-extended semen (Parks and Graham, 1992). When frozen thawed semen is used for insemination it is recommended that the insemination should occur at 4 to $20 \mathrm{hrs}$ from estrus for an acceptable pregnancy rates. It would be interested to test whether the interval from estrus to AI with fresh semen is different from or similar as in insemination with frozen semen. We hypothesize that insemination with fresh extended

semen will improve the AI pregnancy rate due to its prolonged longevity in female reproductive tract compared to frozen thawed semen due to its increased longevity in female reproductive tract. The objective of this trial was to determine the effect of fresh and frozen semen on fixed-time AI pregnancy rate in beef cows synchronized with progesterone based $\mathrm{CO}$ Synch protocols and inseminated at different estrus-AI intervals.

\section{Materials and methods}

Two mature, known fertile Angus bulls were used in this study. Prior to initiation of the study, 0.5 $\mathrm{mL}$ semen samples of each sire were obtained weekly for 3 consecutive weeks and tested negative by culture for presence of Mycoplasma sp. according industry guidelines (Certified Semen Services, Columbia, MO, USA). On collection day two ejaculates were collected serially by artificial vagina and pooled within bull. The pooled semen from each bull was assessed for motility and concentration and then divided into two aliquots.
\end{abstract}




\section{Figure-1. Schematic presentation of CO-Synch-CIDR protocol}

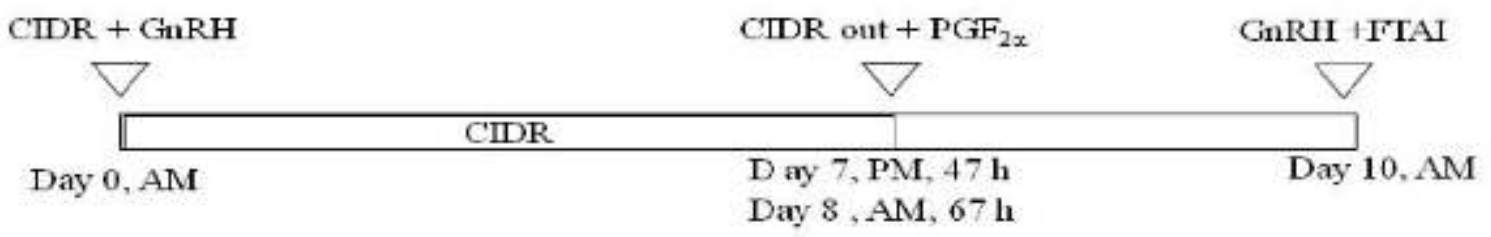

Aliquot one was extended using Caprogen diluent (LIC, Hamilton, New Zealand) to a concentration of 3 $\times 10^{6} \%$ in $0.25 \mathrm{~mL}$ French straw. Fresh semen samples were packaged in $0.25 \mathrm{~mL}$ French straws that are specially designed for use with non-frozen semen (IMV Technologies, L'Aigle, France; reference \# 006937). Straws were stored in Styrofoam boxes at ambient temperature until insemination. Aliquot two was extended using an egg-yolk-glycerol extender to a concentration of $20 \times 10^{6}$ in $0.5 \mathrm{~mL}$ French straw and frozen in liquid nitrogen vapor (-196 C). Semen straws were shipped from the stud center to the breeding locations and cows were artificially inseminated within 48 hours of collection.

Angus cross beef cows $(\mathrm{N}=180)$ were inseminated at $47 \mathrm{~h} \mathrm{AI}$ (early) or 67h (late) from CIDR removal with fresh extended (3 million sperm) or frozen thawed (20 million sperm) semen. All cows were synchronized with CO-Synch-CIDR protocols for a fixed-time AI. Briefly, all cows received $100 \mathrm{~g}$ of gonadorelin diacetate tetrahydrate im (GnRH; Cystorelin sterile solution, Merial, Athens, GA, USA) and a Controlled Internal Drug Release insert (CIDR; Eazi-Breed $^{\mathrm{TM}}$ CIDR cattle insert ${ }^{\circledR}$, Pfizer Animal Health, New York, NY, USA) on day 0. Additionally, body condition scores (BCS) of all cows were recorded on Day 0. The CIDRs were removed and 25 $\mathrm{mg}$ of dinoprost (PGF2; Lutalyse sterile solution, Pfizer Animal Health, New York, NY, USA) was administered im in the evening of day 7 for $67 \mathrm{~h}$ group and in the morning of day 8 for the $47 \mathrm{~h}$ group. Cows were inseminated with either fresh or frozen semen in the morning, on day 10 , and received $100 \mathrm{~g}$ of GnRH concurrently.

Cows randomly assigned to fresh semen and frozen semen groups and AI sires. A subset of cows $(\mathrm{N}=110)$ received a Heatwatch pressure sensor (Heatwatch estrus detection system, CowChips, LLC. Manalapan, NJ. 07726), at CIDR removal for 7 days to determine the time of estrus. (Heatwatch estrus detection system utilizes unique radio frequency technology that catches every cow in heat, recording the date, time and duration of every mount. Utilizing wireless technology, Heatwatch uses small, digital radio transmitters incorporating a pressure switch that are glued onto the tailheads of the cattle. These transmitters continuously monitor all mounting activity of cattle and transmit the mounting data (cow I.D., date of mount, time of day of the mount \& duration of the mount) to the computer where advanced software algorithms examine the mounting profile of each animal. The software is looking for mounting criteria indicative of true estrus. For this study 3 mounting activity within 2 minutes period by a cow was considered as estrus).

Pregnancy diagnosis was performed 60 days after insemination using transrectal ultrasonography (Sonosite 180 plus, Sonosite Inc., Bothell, WA, USA). AI pregnancy rate was calculated by number of cows diagnosed pregnant divided by number of cows inseminated.

Statistical analysis: Mixed model (GLIMMIX procedure of SAS version 9.12, SAS Institute Inc., Cary, NC 27513) was used to determine the effect of time of insemination late $(67 \pm 0.3 \mathrm{~h}$ from CIDR removal) vs. early ( $47 \pm 0.4 \mathrm{~h}$ from CIDR removal) and time interval from estrus to AI (before estrus, 0 to $16 \mathrm{~h}$ after estrus and more than $16 \mathrm{~h}$ after estrus) on AI pregnancy. Other variables included in the model were semen type (Fresh, $3 \times 10^{6}$ vs. Frozen, $20 \times 10^{6}$ ), AI sires (1 vs. 2$)$, BCS (<5, 5 to $6,>6$; 1-emaciated; 9-obese), days postpartum at initiation of synchronization (30 to $60,61$ to $80,>80)$, age of dam $(2,3$ to 6 and $>6)$. $P$ value of 0.05 was considered significant. Model was created by manual backward stepwise elimination procedure ( $\mathrm{P}>0.10$ for exclusion). Only variables of interest and statistically significant variables were retained in the final model.

\section{Results}

Cows inseminated at $67 \mathrm{hr}$. had numerically higher fixed time AI pregnancy compared to cows inseminated at $47 \mathrm{~h}[44.4 \%$ (40/90) vs. $33.3 \%$ (30/90); $\mathrm{P}=0.13$ ]. Cows inseminated with frozen thawed semen had similar fixed time AI pregnancy compared to fresh extended semen [40.8 (31/76) vs. 37.5\% (39/104); 
Figure-2. Mean Al pregnancy rate in beef cows bred with fresh or frozen semen during different interval from estrus to Al

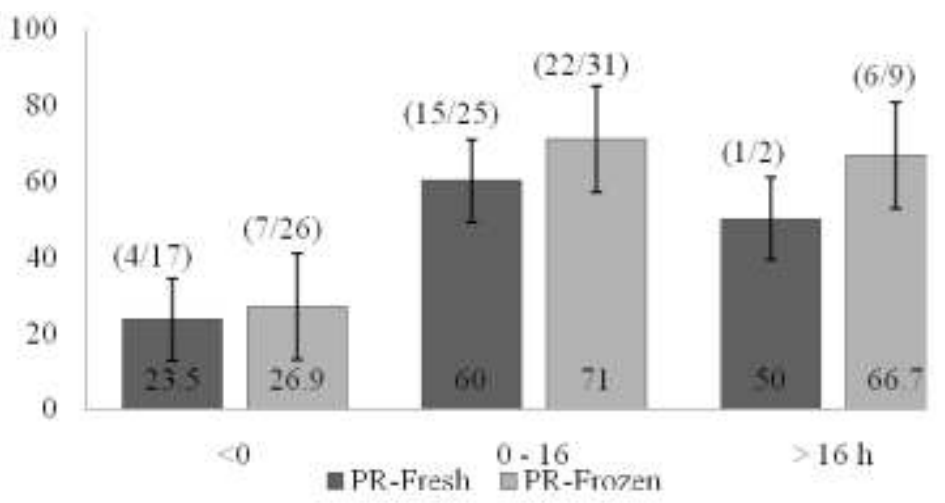

$\mathrm{P}=0.66]$. The AI pregnancy outcome for fresh semen for the 3 estrus-AI intervals was similar to frozen semen (Figure 2). Cows inseminated before estrus had lower AI pregnancy rate compared to cows inseminated after estrus [25.6\% (11/43) vs. 65.7 (44/67); P<0.05; Figure 2].

\section{Discussion}

In the current study, cows inseminated with frozen thawed semen had similar fixed time AI pregnancy compared to fresh extended semen. It is evident that an acceptable pregnancy rate can be achieved with fresh extended semen at a lower sperm number $\left(3 \times 10^{6}\right)$ compared to frozen semen $\left(20 \times 10^{6}\right)$. From clinical and AI standpoint, it is profitable to utilize fresh extended semen for a high demand AI sire instead of frozen semen.

In the current study, there was no difference in AI pregnancy rate for three different estrus-AI intervals tested. It has been documented that there are differences between sires in functional and structural characteristics of post-thaw semen. Some AI sires better withstand insult during the freeze-thaw process compared to other sires. The AI sires used in the study were highly fertile sires. It is possible that the frozen semen form these sires had good post-thaw semen parameters similar to fresh semen parameters. In addition, ovulation time can influence reproductive success when using either fresh or frozen semen (Macmillan etal., 1975).

Insemination with frozen semen $10 \mathrm{~h}$ before projected ovulation decreased conception rate compared with $5 \mathrm{~h}$ before projected ovulation, which may be due to the shorter capacitation time of frozen semen or increased population of precapacitated frozen sperm (Parrish and Foote, 1986). It was evident in the present study that cows inseminated before estrus had lower AI pregnancy rate compared to cows inseminated after estrus. Acceptable AI pregnancy was achievable with AI up to $72 \mathrm{hrs}$ after CIDR removal. Cows inseminated with fresh or frozen semen from 0 to $20 \mathrm{hrs}$ after estrus resulted in at least 50\% AI pregnancy. In conclusion, the fresh semen yielded similar AI pregnancy as frozen semen when inseminated at different estrus-AI interval in a fixed time AI breeding program in beef cows.

\section{Acknowledgement}

The authors thank Select Sires Inc., for providing semen and partial financial support for the study. The authors also extend their gratitude to Virginia Department of Corrections for enrolling their cattle in the study.

\section{References}

1. Parks, J.E., Graham, J.K., (1992). Effects of cryopreservation procedures on sperm membranes. Theriogenology 38, 209-222.

2. Vishwanath, R., Pitt, C.J., Shannon, P., (1996). Sperm numbers, semen age and fertility in fresh and frozen bovine semen. Proc NZSoc Anim Prod 56, 31-34.

3. Macmillan, K.L., Watson, J.D., (1975). Fertility differences between groups of sires relative to the stage of oestrus at the time of insemination. Anim Prod 21, 243-249.

4. Parrish, J.J., Foote, R.H., (1986). Fertility of cooled and frozen rabbit sperm measured by competitive fertilization. Biol Reprod 35, 253-257. 